\title{
Trust Trade-off Analysis for Security Requirements Engineering
}

\author{
Golnaz Elahi \\ Department of Computer Science, University of Toronto \\ gelahi@cs.toronto.edu
}

\author{
Eric $\mathrm{Yu}$ \\ Faculty of Information, University of Toronto, \\ yu@ischool.utoronto.ca
}

\begin{abstract}
Security requirements often have implicit assumptions about trust relationships among actors. The more actors trust each other, the less stringent the security requirements are likely to be. Trust always involves the risk of mistrust; hence, trust implies a trade-off: gaining some bene ts from depending on a second party in trade for getting exposed to security and privacy risks. When trust assumptions are implicit, these trust trade-offs are made implicitly and in an ad-hoc way. By taking advantage of agent- and goal-oriented analysis, we propose a method for discovering trade-offs that trust relationships bring. This method aims to help the analyst select among alternative dependency relationships by making explicit trust trade-offs. We propose a simple algorithm for making the trade-offs in a way that reaches a balance between costs and bene ts.
\end{abstract}

\section{INTRODUCTION}

Complex systems with several requirements and multiple stakeholders typically spread the complexity over interacting and probably distributed actors and subsystems. We implicitly and indirectly trust the complex systems, their subsystems, interacting actors and systems, and their developers to provide the expected services and not abuse their capabilities and permissions. Attacks and vulnerability exploitation are results of wrong assumptions about the trustworthiness of the sources of the attacks, the environment, or solutions. Therefore, trust and trustworthiness are stated as the foundations of security [1].

Relying on any technology or solution requires a careful analysis of the potential abuses of trust. For example, in an indoor WiFi-based navigation system, users estimate their location inside the building by relying on signals that they receive from $\mathrm{WiFi}$ access points and trust that the signals are authentic and reliable. Using pre-installed WiFi access points is economical; however, hostile or compromised WiFi access points may be used to mislead the users.

Mistrust means the trustee has not provided the expected service or abused the permission in an unexpected way [2]. Given the possibility of mistrust, trust involves accepting potential risks in return for some bene ts gained by depending on the another party. Similarly, in psychology studies, trust has been recognized as a bet that implies some risks [3].

Depending on a human, software agent, service provider, or technology implies a trade-off: gaining some bene ts from a second party in exchange for getting exposed to security and privacy risks. These trade-offs are usually made implicitly. This calls for a systematic way for analyzing and making trade-offs that trust relationships impose. This paper proposes an agent- and goal-oriented method for analyzing trust trade-offs. The general idea is to analyze the potential malicious behavior of trustee parties in case they abuse their capabilities and permissions. The method helps decide whether to depend on a trustee party, and to select a party among alternatives. The goal is to make the trust trade-offs in a way that simultaneously satis es multiple requirements of different stakeholders or at least achieves a balance among con icting requirements.

\section{TRust Modeling AND ANALYSis ApproACHES}

The importance of trust has been recognized in several security requirements engineering methods, which focus on analyzing trust relationships and trust assumptions [4], [5], [6], [1]. Viega et al. argue that the basis of trust relationships and trust formation can dramatically affect the underlying security of any system [1].

Approaches such as [6], [7] explicitly capture, model, and analyze trust assumptions. By considering trust assumptions, a requirements engineer believes and accepts that certain properties hold in that domain. The explicit consideration of trust assumptions in the domain model documents the ways in which the analysts choose to trust the behavior of domains and limit the scope of the analysis to these domains that are in the context [6].

Some approaches such as [5], [8] deal with trust as a concern in social and organizational relationships. Giorgini et al. [5] proposed Secure Tropos as a modeling and analysis method for analyzing trust relationships in agent-oriented systems. In this approach, security requirements are analyzed based on the concepts of ownership, permission, delegation, and trust within the normal functional requirements model and actor dependencies. The explicit trust relationship modeling helps analysts make an informed decision about access control rules, permissions, and delegations.

Liu et al. [8] model trustworthiness as an objective of stakeholders which in uences their dependencies and goals satisfaction. In this approach, $\mathrm{i}^{*}$ models are used for reasoning about trustworthiness of the actors and the goal models record the rational for trust assumptions and relationships. The models capture the impact of goals and decisions of stakeholders on trustworthiness. 


\section{TRust TRAde-OFf Modeling}

Goal-oriented requirements engineering helps uncover intentions of users for depending on a software system. Agent orientation along with goal-oriented requirements engineering is useful for understanding software systems, their environments, and users as active components that interact with each other to ful 11 stakeholders' goals. Van Lamsweerde [9] states that goal orientation is useful for analyzing software-to-be and its environment, elaborating requirements, capturing rationale for requirements operationalizations, structuring and documenting requirements, and supporting for exploration of alternatives.

The aim of trust trade-off analysis is to evaluate if the potential risks posed by dependee parties outweigh the bene ts of the dependency relationship; and if so, select an alternative party which offers the best balance between the risks and bene ts of dependencies. The proposed method adopts existing techniques for the rst six steps. The last step of the method, the main contribution of this work, uses the results from the preceding steps for discovering and analyzing trust trade-offs. This section motivates and describes the modeling and analysis activities in each step.

Step One: Identify Actors and Actors' Dependencies.

In the rst step, stakeholders and requirements analysts collaboratively detect and model main actors involved in the system such as humans, sub-systems, software agents, service providers and receivers. The dependency network is expressed by using i* Strategic Dependency (SD) models [10], and later is used to discover the trust relationships and assumptions about the actors.

\section{Step Two: Model and Re ne Actors' Goals.}

In this step, i* SD models are extended to $i^{*}$ Strategic Rationale (SR) models [10], where top goals and softgoals (quality goals) of actors are detected and re ned into nergrained (soft)goals, tasks, and resources. Goal re nement may result in discovering new dependency relations. SR models also capture alternative actors to depend on or alternative ways that the depender actor achieve its goals. $i^{*}$ contribution links are used to model the bene ts of dependencies (i.e., positive impacts on satisfaction of (soft)goals).

Step Three: Discover and Model Trust Relationships in the Dependency Chain.

Giorgini et al. [5] assert that task, goal, and resource dependencies indicate an implicit "trust" and "delegation of execution". By trust of execution and delegation of execution, we mean that the delegator (truster) depends on the delegatee (trustee) party to provide the requested service or resource [5]; therefore, the delegatee (trustee) thinks I have permission to provide this service. However, dependency relationships do not necessarily imply a trust relationship. In some cases, an actor must delegate a service to an untrusted party, or the potential risks that the dependee may bring outweigh the bene ts of the dependency relationship. Therefore, Giorgini et al. [4] propose an explicit distrust relationship in the actors' dependency modeling, where distrust means one party does not trust another [2].

In this step, the requirements analyst questions if stakeholders and security experts trust (or distrust) the alternative dependee actors, and models these relationships explicitly. Figure 1(b) and (c) depicts different interpretations and modeling notation of dependency relationships into explicit trust, distrust [4], and delegation of execution relationships. A resource dependency implicitly indicates another type of trust and delegation relation. In a resource dependency, the dependee also trusts and delegates the permission to use the resource to the truster. Similar to the previous discussion, the dependee does not necessarily trust the depender actor, which can be captured by distrust relationship.

Step Four: Recording Trust Rationale.

In this step, stakeholders describe their rationales for trusting or distrusting a party. In psychology studies, (dis)trust is viewed as a mental state based on a set of beliefs, goals, and a decision [3]; therefore, we use the $i^{*}$ "belief" element for recording the rationale of (dis)trusting an actor from the viewpoint of the depender actor. These rationales are related to the (dis)trust relationship as shown in the example in Figure 1(a).

Example: Figure 1(a) shows a part of the actors' dependency model, their (dis)trust relationships, and rationale for the (dis)trust assumptions for the WiFi navigation case system. In this model, the Client provides the WiFi signal information to the Server and gives the permission to the server to use this data. The developers of the system assert that due to the open source development of the server service, the trust relationship between the client and the server is justi able.

Step Five: Replace the Trustee Party with a Corresponding Malicious Party.

The underlying idea for trust trade-off analysis in this paper is obtained from the security and privacy requirements analysis method proposed by Liu et al. [11]. Liu et al. assert that dependency relationships bring risks to the system and depender actors; therefore the dependee actors are substituted with the corresponding attacker. The main purpose of this step is to analyze how capabilities and permissions granted to the trustee can be abused for malicious purposes. While traditional security risk analysis methods consider probabilities of threats and nancial costs, in our approach, risks are analyzed in terms of negative impacts of attacks on stakeholders' goals such as security, privacy, usability, and nancial costs.

The malicious actor inherits capabilities, permissions, and resources to which the dependee is delegated and granted. The main purpose of this step is to analyze how capabilities and permissions granted to the dependee can be abused for malicious purposes. In this step, the requirements analyst brainstorms all the possible ways that the dependee party can abuse the permissions and capabilities. The general idea 


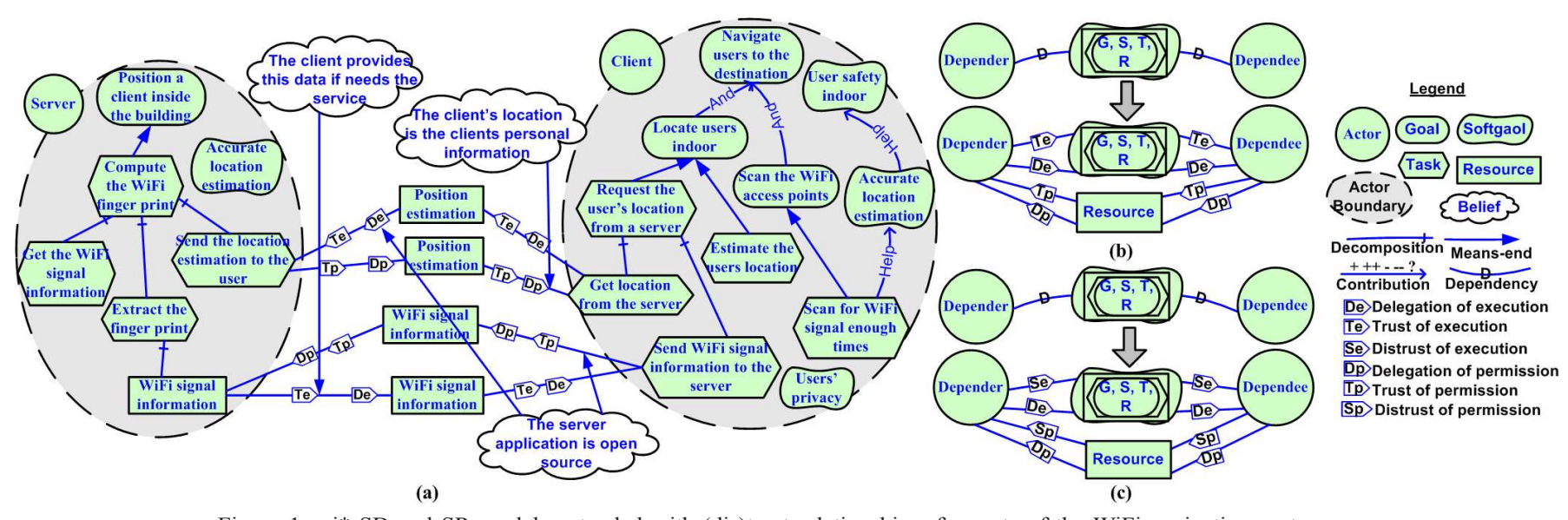

Figure 1. i* SD and SR models extended with (dis)trust relationships, for parts of the WiFi navigation system.

is that each task and resource of the trustee can be abused for malicious purposes. We adopt security extensions to the $i^{*}$ modeling notation [12] for modeling malicious behavior of dependee actors as a malicious counterpart.

Example: In Figure 2(a), the Server is substituted with its malicious counterpart. In this model, the malicious server can Monitor movement of users in buildings, and Reveal the users' positions to other parties. The malicious server relies on the capabilities and permissions of the Server such as having access to WiFi signal information or Sending an incorrect location estimation to the user. The risks of the trust relationship are modeled as negative contributions of malicious server's tasks on the Client's softgoals.

\section{Step Six: Model and Analyze Vulnerabilities.}

A trust relationship may not pose risks when the depender actor fully trusts the dependee and the dependee behaves in the expected manner. However, trust trade-off analysis is not limited to the issue of abuse by the trustee. In addition to the possibility of mistrust, depending on a software, human, technology, or service provider may bring weaknesses (vulnerabilities) from the vulnerable dependee to the boundary of the depender actor. The term vulnerability is used here in the security engineering sense, i.e., systemrelated defects and weaknesses such as buffer over ow, hostile user's inputs, and malware that can be exploited by an attacker to compromise the system.

In this step, the vulnerabilities that the dependee parties bring to the system are added to the goal model using the notation proposed in [13]. Potential attacks that exploit the vulnerabilities are detected, and effects of the vulnerabilities' exploitation are related to the goals and tasks of the depender parties. Through goal model evaluation [14], [13] (in the next step), ultimate effects of vulnerabilities on (soft)goals satisfaction are propagated to the top (soft)goals of the actors. Although the effects of the vulnerabilities are propagated by the goal model evaluation, distribution of the vulnerabilities in the dependency network helps identify different vulnerabilities' exploitations and different effects in the context of other actors, resources, or tasks.
Example: Figure 2(b) shows the vulnerabilities that the WiFi Access Point brings to the system. Broadcasting a signal by the WiFi Access Point introduces vulnerabilities such as Termination of the signal and Corruption of the signal. The Termination of the signal has negatives effects on Portability of users inside buildings. The Corruption of the signal gets propagated to the Client actor and has negative effects on Accurate location estimation. The vulnerabilities can get exploited by attacks of an Outsider Attacker; for example, the attacker can Manipulate the broadcasted WiFi signal with a hardware to Mislead the clients.

\section{Step Seven: Analyze the Trust Trade-offs.}

Once alternative solutions have been suf ciently elaborated, and potential risks, vulnerabilities, and negative and positive contributions of dependency relationships are explicitly expressed in the goal model, costs and bene ts and trade-offs of the alternative dependency relationships can be uncovered. These costs and bene ts are not necessarily nancial, but are positive and negative impacts on goals and softgoals of the actors. The costs of a dependency relationship are risks that the malicious dependee poses and vulnerabilities that threaten security requirements; at the same time, the depender gains bene ts by depending on another actor. In this step, by using goal model evaluation techniques [15], [14], the costs and bene ts of each alternative dependee are evaluated in terms of satisfaction or denial of top (soft)goals of the depender.

Evaluating a goal model for an alternative generates a model with visual evaluation labels which graphically demonstrates advantages and disadvantages of the alternatives. The evaluation is repeated for the corresponding malicious dependee model to evaluate the risks of the dependency relationship. In order to compare several alternatives, one needs to evaluate the goal model for every alternative solution, which results in several goals models with different visual evaluation labels distributed in the graph. Because of humans' limited visual perception, comparing several graphical results of goal model evaluation is challenging. To improve the representation schema for decision making, we 


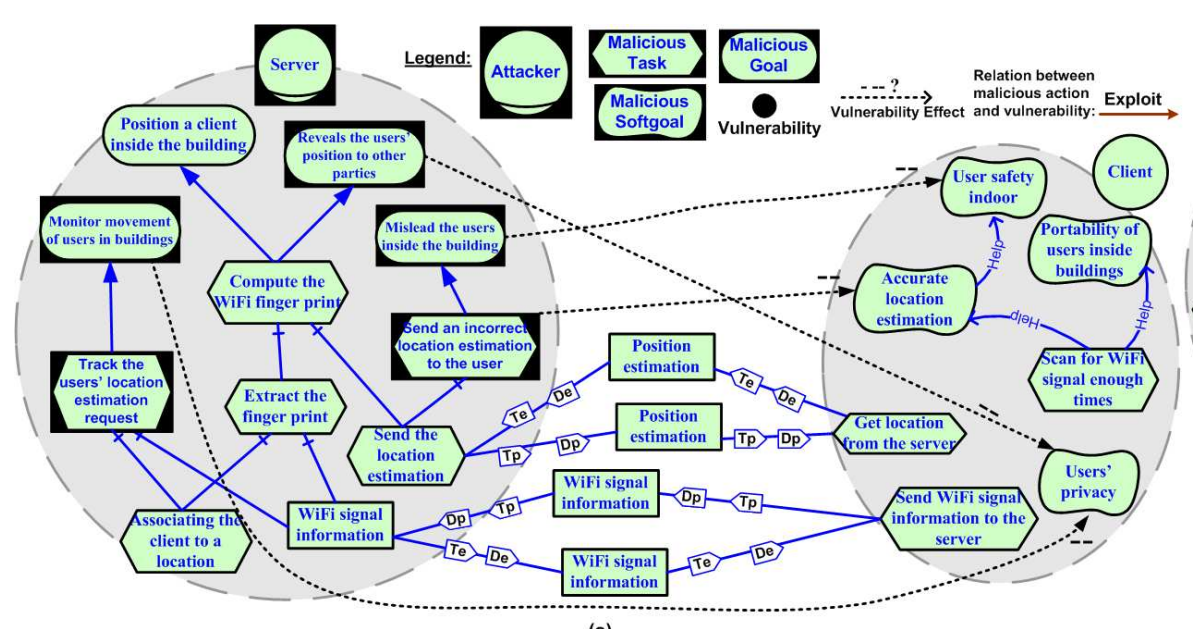

(a)

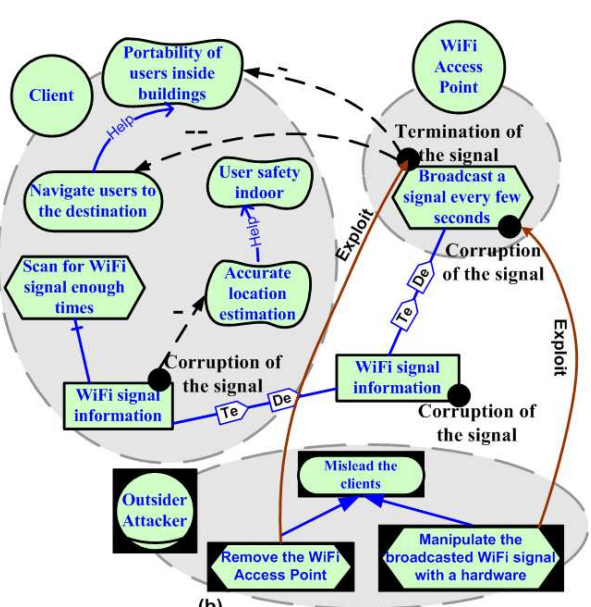

Figure 2. Malicious (trustee) server and vulnerabilities that outside attacker exploit, for parts of the WiFi navigation system.

Table I

TRUST TRADE-OFF TABLE (FOR THE WIFI NAVIGATION SYSTEM) ${ }^{1}$

\begin{tabular}{|c|c|c|c|c|}
\hline Alternatives & $S_{1}$ & $S_{2}$ & $S_{3}$ & $S_{4}$ \\
\hline Depending on WiFi signals & PS & PS & PS & $\mathrm{FS}$ \\
\hline $\begin{array}{l}\text { Depending on WiFi signals } \\
\text { (Malicious trustee) }\end{array}$ & FD & PD & PD & FS \\
\hline Depending on RFID & $\mathrm{FS}$ & PS & PS & FD \\
\hline $\begin{array}{l}\text { Depending on WiFi signals } \\
\text { (Malicious trustee) }\end{array}$ & PD & PD & PS & FD \\
\hline
\end{tabular}

propose organizing results evaluation labels into a table that lists and compares costs and bene ts of alternatives. Similar tables have been suggested in [11] for decision making based on goal model evaluation results, and in ATAM and CBAM for architecture trade-off analysis [16].

Example: In the WiFi-based navigation system, suppose the client can either depend on RFID tags that need to be installed inside the building or depend on WiFi signals, and the Client's softgoals are Portability of users inside buildings $\left(S_{1}\right)$, Accurate location estimation $\left(S_{2}\right)$, Safety $\left(S_{3}\right)$, Low cost of signal broadcasting devices $\left(S_{4}\right)$. Table 1 shows a part of the trust trade-off table for the Client's softgoals.

Table 1 lists alternative ways to achieve goals of actors in the rows. The columns record the criteria for comparing alternatives (i.e., the top goals and softgoals of the depender actor). Each cell of the table records evaluation label of a (soft)goal achieved by an alternative. For each alternative, the corresponding evaluation labels in case of mistrust are recorded in another row. In this way, the table compares costs (risks) and bene ts of alternative dependency relationships.

\section{Trade-OfF ANAlysis For SECURITy REQUIREMENTS ENGINEERING}

The ultimate goal of trust trade-off analysis is to decide about depending on a party or selecting a dependee among alternatives. The rst part of this section argues why goal

\footnotetext{
${ }^{1}$ PS indicates Partially Satis ed; FS means Fully Satis ed; PD means Partially Denied, and FD indicates Fully Denied.
}

modeling helps analyze trade-offs. The second part of the section presents a simple multi-criteria decision analysis algorithm for making trust trade-offs.

\section{A. Goal Modeling for Trade-off Analysis}

By developing a goal model, the criteria for comparing alternatives are interconnected and re ned into ne-grained softgoals. Goal models also document the reasons an alternative is preferred to the others in terms contributions of alternatives on softgoals. As the interconnections among criteria and alternatives grow into a complicated graph, goal model evaluation methods help draw conclusions about nal costs and bene ts of each alternative.

Structuring the trade-off decision problem into goal models helps re ne and resolve the trade-offs among criteria of comparison. We explain this claim by an example. In the model in Figure 3(a), by adopting either the alternative $T_{1}$ or $T_{2}$, one of the $S_{1}$ or $S_{2}$ softgoals is satis ed and the other one is denied. The only way to resolve the trade-off between $S_{1}$ and $S_{2}$ is to prioritize the softgoals. If the stakeholders are not able to trade one softgoal for the other, this situation is a dead-end decision making case. However, prioritizing the requirements is not always suf cient to make the tradeoffs. For example, if $S_{1}$ is preferred to $S_{2}$ (Figure 3(b)), the stakeholder may still not agree with full denial of a less important criterion $\left(S_{2}\right)$ in exchange for partial satisfaction of the higher-priority softgoal $\left(S_{1}\right)$.

We argue that re ning the softgoals and alternatives may help make a trade-off in such conditions. Figure 3(c) shows the re nement of the goal model in Figure 3(a). In this model, the softgoals are re ned to ner-grained softgoals, and $T_{2}$ is decomposed into more detailed tasks. By re ning softgoals, the model shows why the abstract softgoals are in trade-off. For example, $S_{4}$ and $S_{5}$ are in trade-offs (which causes the trade-off between $S_{1}$ and $S_{2}$ ultimately), and $S_{3}$ and $S_{6}$ can be satis ed simultaneously. The re nement of alternatives uncovers what subtasks result in trade-offs. For 


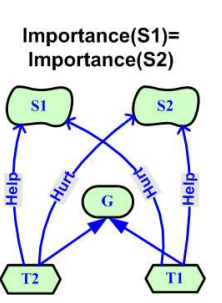

(a)

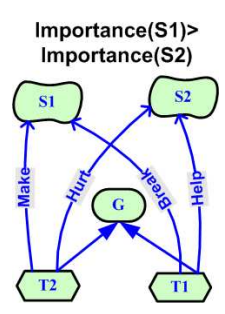

(b)



(c)
Figure 3. A trade-off example and resolving the trade-off through goal re nement

example, performing $T_{3}$ does not introduce any trade-off, while $T_{4}$ causes $S_{5}$ be denied.

In Figure 3(c), the stakeholder is not limited to trade $S_{1}$ for $S_{2}$, but also can trade $S_{4}$ for $S_{5}$ by performing $T_{3}$ and $T_{1}$. By re ning the criteria of comparison and alternatives, stakeholders have more options to make the trade-offs. Therefore, the stakeholder is not limited to choose between two highlevel goals.

\section{B. Multi-Criteria Decision Analysis for Making Trust Trade- offs}

Once the alternative solutions and softgoals are suf ciently re ned, applying goal model evaluation techniques show how a solution satis es some quality goals and has negative effects on satisfaction of other requirements. This situation results in trade-offs between the positively- and negatively-affected requirements, and calls for a way to select an alternative when suf cient number of alternatives is examined.

In approaches such as Multi-Attribute Utility Theory (MAUT) [17], deciding on alternatives mainly involves assigning importance weights to requirements (i.e., criteria of comparison) and calculating a utility value for each alternative; then the alternative with highest utility is selected. However, quantitative decision analysis methods are not suitable in the context of trust trade-off analysis in early requirements engineering phases, mainly because: 1) Security trade-offs are naturally subjective and security decisions are based on personal judgment and feeling [18] of stakeholders and analysts. 2) Different stakeholders have different expectation from the software system, as well as different level of risk tolerance and personal privacy expectations; therefore, they impose different security requirements which may con ict with security and other (soft)goals of other stakeholders. 3) Analyzing costs and bene ts of security-critical systems may involve ethical issues. In some contexts, quanti cation of human loss or privacy violation is not ethically acceptable [19]. 4) Many quantitative data elicited from stakeholders are imprecise, uncertain, or ill-de ned, and there is a real risk of giving them unwarranted signi cance [20].

To avoid the discussed issues, we take a qualitative decision analysis approach. To select a solution among alternatives in the trust trade-offs table we adapt the "Even Swaps" method [21] which works based on a selective pair-wise
Table II

TRUST TRADE-OFF TABLE WITH $S^{\prime}$ AND $G^{\prime}$ CRITERIA

\begin{tabular}{|l|c|c|c|c|c|}
\hline Alternatives & $S_{1}$ & $S_{1}^{\prime}$ & $S_{3}^{\prime}$ & $S_{4}$ & $S_{4}^{\prime}$ \\
\hline Depending on WiFi signals & $\mathrm{PS}$ & $\mathrm{FD}$ & $\mathrm{PD}$ & $\mathrm{FS}$ & $\mathrm{FS}$ \\
\hline Depending on RFID & $\mathrm{FS}$ & $\mathrm{PD}$ & $\mathrm{PS}$ & $\mathrm{FD}$ & $\mathrm{FD}$ \\
\hline
\end{tabular}

TRUST TRADE-OFF TABLE, WHERE $S_{1}$ CAN BE ELIMINATED

\begin{tabular}{|l|c|c|c|c|c|}
\hline Alternatives & $S_{1}$ & $S_{1}^{\prime}$ & $S_{3}^{\prime}$ & $S_{4}$ & $S_{4}^{\prime}$ \\
\hline Depending on WiFi signals & $\mathrm{FS}$ & $\mathrm{FD}$ & $\mathrm{PD}$ & $\mathrm{FS}$ & $\mathrm{PS}$ \\
\hline Depending on RFID & $\mathrm{FS}$ & $\mathrm{PD}$ & $\mathrm{PS}$ & $\mathrm{FD}$ & $\mathrm{FD}$ \\
\hline
\end{tabular}

comparison of criteria. The criteria of comparison are the goals and softgoals of stakeholders. Qualitative evaluation labels of the (soft)goals indicate the evaluation value for the criteria.

We need to adapt the Even Swaps decision analysis method to incorporate the consequences of the mistrust condition for the trust trade-off analysis. Therefore, for every criterion such as softgoal $S$ or goal $G$, the criterion in mistrust condition, $S^{\prime}$ or $G^{\prime}$, is added to the table. In this way, the alternatives are compared based on their costs and bene ts both in the expected and mistrust situations. In the Even Swaps method, the size of the problem is incrementally reduced by applying two kind of steps iteratively to eliminate alternatives (rows) that are clearly worse than (dominated by) the others, and to eliminate criteria (columns) that do not differentiate the alternatives. For example, in Table 1, $S_{2}, S_{2}^{\prime}$, and $S_{3}$ do not have any impact on the comparison and can be removed from the table (Table 2).

To eliminate further columns, we take two alternatives that have different evaluation levels for some criterion. We ask the stakeholders to give up some ground on that criteria in return for gains on another criterion. By making two alternatives even with respect to some criterion (i.e., achieved to the same extent), we can eliminate that criterion from further consideration. For example, in Table 2, depending on "WiFi signals" partially satis es $S_{1}$ and depending on "RFID" fully satis es $S_{1}$. One can improve the softgoal $S_{1}$ to FS for the rst alternative, but in return, we need to ask the stakeholders how much another attribute should be reduced if $S_{1}$ is improved to FS. For example, the stakeholder may be able to trade $S_{4}^{\prime}$ being PS for $S_{1}$ being FS. The result of this swapping is shown in Table 3 . The highlighted column $\left(S_{1}\right)$ can be eliminated from the analysis.

The rest of the comparison continues with new evaluation values assigned to the criteria until an alternative has better evaluation labels for all of the remained criteria and is selected. The swapping process might be in uenced by the priorities of the criteria for the stakeholders, the strength of the trust relationship, and rationales for trustworthiness of a party. However, the method will work if one is able to nd two criteria for which a swap is possible.

\section{Conclusions, Limitations, And Future Work}

This paper argues that implicit (dis)trust in dependency relationships needs to be explicitly analyzed as a trade-off. 
We proposed a systematic way for discovering trade-offs that dependency relationships impose. The proposed method takes advantage of agent- and goal-oriented modeling for structuring requirements of different stakeholders along with alternative solutions or parties that stakeholders can depend on. We adapted the Even Swaps multi-criteria decision making method for selecting an alternative among others. Our research method included several case studies developed in collaboration with requirements and system analysts with limited security knowledge (e.g., WiFi-based navigation system developed at Adaptive Technology Resource Centre at the University of Toronto, SlashID authentication protocol [22]). Based on the result of the case studies and interviewing the analysts and modelers involved in the case studies, the modeling and analysis method was revised and improved.

Further work is required to evaluate, strengthen, and expand the proposed method. The goal models extended with trust relationships, vulnerabilities, and malicious behavior do not scale effectively. We need to develop tools for model management, semi-automated transformation of models, and displaying different views of the models. We discussed that structuring the trade-off problem into goal models and re ning higher level tasks and softgoals may help resolve the trade-offs. This claim needs to be examined in more case studies and empirical examples. We also argued that gathering rationales for (dis)trust relationships guide stakeholders to evaluate the risk of mistrust and select the most costeffective alternative. This claim needs to be supported with empirical evidences showing that collection and analysis of the rationales improve the decision making quality.

Currently, the Even Swaps method focuses on balancing the requirements of a single stakeholder. We need to improve the decision analysis method for balancing the requirements of multiple stakeholders simultaneously. In future work, we need to incorporate the stakeholders preferences into the decision making method as well. We also need to extend the method for incorporating the (dis)trust rationale and available probabilities of mistrust into the Even Swaps decision making process. Finally, Even Swaps method does not offer a way for selecting the attributes to swap; hence, in future work, we look for a suitable order for eliminating criteria from the trade-off table.

Acknowledgments. Financial support from the Natural Science and Engineering Research Council of Canada and Bell University Labs is gratefully acknowledged. The authors thank Jorge Silva at the Adaptive Technology Resource Centre, University of Toronto for the opportunity of collaboration, and Jocelyn Simmonds for useful insights on the decision making algorithm.

\section{REFERENCES}

[1] J. Viega, T. Kohno, and B. Potter, "Trust (and mistrust) in secure applications," Commun. ACM, vol. 44, no. 2, pp. 3136,2001
[2] S. P. Marsh and M. R. Dibben, "Trust, untrust, distrust and mistrust - an exploration of the dark(er) side," vol. 3477, pp. 17-33, 2005.

[3] C. Castelfranchi and R. Falcone, "Principles of trust for MAS: cognitive anatomy, social importance, and quanti cation," in Proc. of Multi Agent Systems, 1998, pp. 72-79.

[4] P. Giorgini, F. Massacci, and N. Zannone, "Security and trust requirements engineering," in Proc. of FOSAD, 2005, pp. 237-272.

[5] P. Giorgini, F. Massacci, J. Mylopoulos, and N. Zannone, "Modeling security requirements through ownership, permission and delegation," in Proc. of RE'05, 2005, pp. 167-176.

[6] B. Haley, C. Laney, D. Moffett, and B. Nuseibeh, "Using trust assumptions with security requirements," Requir. Eng., vol. 11, no. 2 , pp. 138-151, 2006.

[7] C. Haley, R. Laney, J. Moffett, and B. Nuseibeh, "Security requirements engineering: A framework for representation and analysis," TSE, vol. 34, no. 1, pp. 133-153, 2008.

[8] E. S. K. Yu and L. Liu, "Modelling trust for system design using the $\mathrm{i}^{*}$ strategic actors framework," in Proc. of the workshop on Deception, Fraud, and Trust in Agent Societies, 2001, pp. 175-194.

[9] A. van Lamsweerde, "Requirements engineering in the year 00: a research perspective," in Proc. of ICSE'00, 2000, pp. 5-19.

[10] E. Yu, "Modeling Strategic Relationships for Process Reengineering," Ph.D. dissertation, University of Toronto, 1995

[11] L. Liu, E. Yu, and J. Mylopoulos, "Security and privacy requirements analysis within a social setting," in Proc. of $R E^{\prime} 03,2003$, p. 151.

[12] G. Elahi and E. Yu, "A goal oriented approach for modeling and analyzing security trade-offs," in Proc. of ER'07, ser. LNCS 4801, 2007, pp. 375-390.

[13] G. Elahi, E. Yu, and N. Zannone, "A vulnerability-centric requirements engineering framework: Analyzing security attacks, countermeasures, and requirements based on vulnerabilities," Manuscript submitted to REJ, 2009.

[14] J. Horkoff and E. Yu, "A Qualitative, Interactive Evaluation Procedure for Goal- and Agent-Oriented Models," in CAiSE Forum. CEUR Workshop Proceedings, 2009.

[15] L. Chung, B. A. Nixon, E. Yu, and J. Mylopoulos, NonFunctional Requirements in Software Engineering. Kluwer Academic, 1999.

[16] L. Bass, P. Clements, and R. Kazman, Software Architecture in Practice. Boston, MA, USA: Second Edition, Addison Wesley, 2003.

[17] R. L. Keeney and H. Raiffa, Decisions with multiple objectives : preferences and value tradeoffs. Wiley, 1976.

[18] B. Schneier, Beyond Fear. Springer, 2003.

[19] B. Blakley, E. McDermott, and D. Geer, "Information security is information risk management," in Proc. of NSPW '01, 2001, pp. $97-104$.

[20] J. Figueira, S. Greco, and M. Ehrgott, Multiple Criteria Decision Analysis: State of the Art Surveys. Springer Verlag, 2005.

[21] J. S. Hammond, R. L. Keeney, and H. Raiffa, Smart choices : a practical guide to making better life decisions. Broadway Books, 2002.

[22] G. Elahi, Z. Lieber, and E. Yu, "Trade-off analysis of identity management systems with an untrusted identity provider," in Proc. of COMPSAC'08, STPSA'08 Workshop, 2008, pp. 661666. 